\title{
Fulminant infection by uncommon organisms in animal bite wounds
}

\begin{abstract}
J K Dutta
Summary

In 1995 and 1996, 215 patients exposed to different species of animals were treated at the Amarnath Polyclinic, Balasore, in India. Among them were two children infected by uncommon organisms, ie, Capnocytophaga canimorsus and Pasteurella multocida; the patients recovered with appropriate antibiotic therapy.
\end{abstract}

Keywords: Capnocytophaga canimorsus; Pasteurella multocida; animal bites

Animal bites are extremely common in developing countries. Infection by uncommon organisms are hardly ever reported from these regions due primarily to the nonavailability of adequate laboratory facilities and lack of awareness among physicians and microbiologists.

Between 1995 and 1996, 215 persons were treated for exposure to different species of animals under the author's care. Two interesting cases of infection following dog bite by uncommon organisms were observed. The case histories are presented and relevant literature is briefly reviewed.

\section{Case reports}

Case 1

A 4-year-old girl was bitten by a stray dog near her house in a semi-urban area. She received severe lacerated wounds on her right cheek before she being rescued by her mother who was bitten on the left cheek. The child attended the local hospital where the wounds were dressed and a dose of tetanus toxoid was administered. She also received a dose of purified chick embryo cell vaccine intramuscularly in the arm on the day of the bite which was continued for a full course of five doses. Penicillin was avoided as she was known to be allergic to penicillin and sulfa drugs. Her parents did not endorse the parenteral administration of any drug, so she was treated with oral erythromycin.

After 2 weeks the wound had not healed adequately and there was recurrent serosanguinous discharge from the area. She developed fever accompanied by chill (temperature $39^{\circ} \mathrm{C}$ ). Some petechiae were observed on the trunk and extremities and mucosal ulceration was noted in the oral cavity. Leucocyte count was $7.1 \times$ $10 \%$ (neutrophils $45 \%$ ). There was moderate thrombocytopenia. A local microbiologist was consulted and on his advice a blood sample was tested in a sophisticated laboratory at a tertiary referral centre. The history of dog bite was given and a request made to look for uncommon organisms. Gram-negative bacilli were isolated after culture which were identified as Capnocytophaga canimorsus. The child was successfully treated with co-amoxiclav to which the organisms were sensitive.

\section{Case 2}

A 5-year-old boy was bitten by a stray dog in his village. He received multiple lacerated wounds with perforation of the left cheek. The child was immediately taken to the nearest hospital where the wounds were cleaned, dressed and seven primary sutures were applied. No prophylactic measures against rabies were initiated at this stage. Two days later, he came under the author's care and was advised to commence passive prophylaxis with rabies immune globulin, which the parents could not afford. Six doses of purified Vero cell rabies vaccine (Verorab: Pasteur Merieux, Lyon, France) alone were administered intramuscularly in the arm, as recommended by the World Health Organisation. ${ }^{1} \mathrm{He}$ was found to be allergic to penicillin and hence he was empirically treated with erythromycin suspension orally.

On removal of the sutures, the wounds had not healed adequately. There was cellulitis of surrounding soft tissues and regional lymph nodes were inflamed. He developed a fever $\left(39.2^{\circ} \mathrm{C}\right)$ and remained in distress. Total leucocyte count was $10.2 \times 10^{9} / 1$ (neutrophils $54 \%$ ). Samples of blood and pus from the wounds were tested in a well-equipped laboratory where Gram-negative coccobacilli, later identified as Pasteurella multocida, were isolated from both specimens after culture. The isolates were sensitive to penicillin and cefotaxime. $\mathrm{He}$ was treated with cefotaxime on which fever subsided and the wounds healed quickly.

\section{Discussion}

Animal bites are frequent among children, who often approach animals for petting but are unable to defend themselves when attacked. In the worst case, mammalian bites may cause soft tissue damage in which fulminant infection by rabies virus and tetanus bacillus may develop. Occasionally, less common fastidious organisms which are commensals in the oral cavity of cats and dogs may infect these wounds. Unless specifically looked for, the diagnosis may be missed in a microbiological laboratory, where they may be regarded as contaminants.

Capnocytophaga species are a group of gliding bacteria which are facultatively 
anaerobic, capnophilic, and Gram-negative. $C$ canimorsus is a normal resident of the canine mouth. Their growth in blood culture is slow but they may be isolated by Gram staining of a buffy coat preparation. The correct diagnosis of infection with this organism in case 1 was possible when the microbiologist was alerted. Usually, infection is associated with asplenism, ${ }^{2}$ hairy cell leukaemia, ${ }^{3}$ liver cirrhosis, ${ }^{4}$ and other immune suppressive states. This organism, designated a Dysgonic Fermenter 2 (DF2) by the US Centers of Disease Control, has caused fulminant bacillaemia and sepsis in some asplenic patients. ${ }^{56}$ Cutaneous infection causing eschar formation and gangrene associated with DF2 septicaemia has also been reported. ${ }^{5}$ Infection by this microbe in healthy subjects is occasionally encountered. ${ }^{8}$ The child in this report had no known risk factors.

Pasteurella multocida is a small non-motile, Gram-negative, bipolar coccobacillus which often inhabits the oropharynx of cats, dogs and

1 World Health Organisation (WHO). Expert committee on rabies. Technical report series 824 . Geneva: WHO, 1992.

2 Dire DJ, Hogan DE, Riggs MW. A prospective evaluation of risk factors for infection from dog bite wounds. Acad Emerg Med 1994;1:258-66.

3 Nodon JA. Capnocytophaga canimorsus septicaemia caused by a dog bite in a hairy cell leukemia patient. $\mathcal{f}$ Clin Microbiol 1992;30:211-13.

4 Roblot P, Bazillou M, Grollier G, Beeg-Giraudon B, Fanchere JL. Septicaemia due to Capnocytophaga canimorsus after a dog bite in a cirrhotic patient. Eur $\mathcal{f}$ Clin Microbiol Infect Dis 1993;12:302-3.

5 Findling JW, Pohlmann GP, Rose HD. Fulminant gram negative bacillemia (DF 2) following a dog bite in an negative bacillemia (DF 2) following a dog

6 Martone WJ, Zuehe RW, Minson GE, Scheld WM. Post 6 Martone WJ, Zuehe RW, Minson GE, Scheld WM. Post
splenectomy sepsis with DF 2: report of a case with isolation some birds as a commensal. Infection by the organism has been identified in cases of Ludwig's angina, epiglottitis, and tonsillitis following animal bites. Fulminant infection caused septicaemia and peritonitis in a cirrhotic cock trainer, ${ }^{9}$ and empyema in another patient. ${ }^{10}$ These organisms have occasionally been isolated from throats of healthy humans with frequent exposure to animals. Invasive pasteurellosis may cause meningitis, pneumonia, and otitis media, due to the spread of organisms from pre-existing colonies in the upper respiratory tract. In the present report, case 2 was effectively treated with cefotaxime, although a cheaper drug like penicillin could have controlled the infection but for his sensitivity. In animal bite wounds initial therapy with penicillin is preferable unless contraindicated, as it can control infection with several microbes including Pasteurella and Capnocytophaga species.

of the organisms from the patient's dog. Ann Intern Med 1980;93:457-8

7 Kalb R, Kaplan MH, Fenenbaum MJ, Joachim GR, Samuels $\mathrm{S}$. Cutaneous infection at dog bite wounds associated with fulminant DF2 septicaemia. Am F Med 1985;78:68790.

8 Hastson P, Gautier PF, Vekemans MC, et al. Fatal capnocytophaga canimorsus septicaemia in a previously healthy woman. Ann Emerg Med 1991;20:93-4.

9 Patton F, Dumas M, Cannon NJ. Pasteurella multicida septicemia and peritonitis in cirrhotic cock trainer with a pet pig. $N$ Engl f Med 1980;303:1126-7.

10 Neison SC, Hammert GS. Pasteurell multicida empyema: case report and review of literature. Am $\mathfrak{f}$ Med Sci $1981 ; 281$ : 43.

Department of Histopathology, Bradford Hospitals NHS Trust, Bradford, BD9 6RJ, UK

P A Batman

\section{Department of}

Obstetrics and

Gynaecology,

Dewsbury \& District

Hospital, Dewsbury,

WF13 4HS, UK

J Thomlinson

V C Moore

West Yorkshire

Analytical Services,

Wakefield, WF1 2TN, UK

R Sykes

Accepted 10 March 1998

\title{
Death due to air embolism during sexual intercourse in the puerperium
}

\author{
P A Batman, J Thomlinson, V C Moore, R Sykes
}

\section{Summary \\ We describe the cases of two young women who died due to air embolism during sexual intercourse early in the puerper- ium.}

Keywords: air embolism; sexual intercourse; puerperium

The review of maternal deaths covering the period 1967-1993 includes over 20 million pregnancies; 18 deaths were due to air embolism. ${ }^{1}$ We describe two such cases occurring within a 2-year period in a West Yorkshire town with approximately 2400 deliveries a year at the local hospital.

\section{Case 1}

A 22-year-old woman was engaged in sexual intercourse with her husband in a rear entry (knee-chest) position 8 days following the spontaneous vaginal delivery of their third child, when she collapsed suddenly and died, passing blood per vagina. Autopsy showed features of air embolism. Air bubbles were present in major vessels of organs inspected in situ, including cerebral and coronary arteries. Frothy blood was detected between the trabeculae in the wall of the right ventricle. The endometrial cavity contained blood clot and fresh haemorrhage, and histological examination of the placental bed showed few thrombosed blood vessels. Most vessels in the placental bed myometrium contained fresh blood and some showed organising luminal thrombus. Post-mortem blood showed an amphetamine concentration of $175 \mathrm{ng} / \mathrm{ml}$.

\section{Case 2}

A 29-year-old woman died suddenly during sexual intercourse with her husband in the 